\title{
A Novel Rule Weighting Approach in Classification Association Rule Mining
}

\author{
Yanbo J. Wang ${ }^{1}$, Qin Xin ${ }^{2}$, and Frans Coenen ${ }^{1}$ \\ ${ }^{1}$ Department of Computer Science, The University of Liverpool \\ Ashton Building, Ashton Street, Liverpool, L69 3BX, United Kingdom \\ \{jwang,frans\}@csc.liv.ac.uk \\ ${ }^{2}$ Department of Informatics, The University of Bergen \\ P.B.7800, N-5020 Bergen, Norway \\ xin@ii.uib.no
}

\begin{abstract}
Classification Association Rule Mining (CARM) is a recent Classification Rule Mining approach that builds an Association Rule Mining based classifier using Classification Association Rules (CARs). Regardless of which particular CARM algorithm is used, a similar set of CARs is always generated from data, and a classifier is usually presented as an ordered CAR list, based on a selected rule ordering strategy. In the past decade, a number of rule ordering strategies have been introduced that can be categorized under three headings: (1) support-confidence, (2) rule weighting, and (3) hybrid. In this paper, we propose an alternative rule-weighting scheme, namely CISRW (Class-Item Score based Rule Weighting), and develop a rule-weighting based rule ordering mechanism based on CISRW. Subsequently, two hybrid strategies are further introduced by combining (1) and CISRW. The experimental results show that the three proposed CISRW based/related rule ordering strategies perform well with respect to the accuracy of classification.
\end{abstract}

\section{Introduction}

Classification Rule Mining (CRM) [12] is a wellknown Data Mining technique for the extraction of hidden Classification Rules (CRs) from a given database that is coupled with a set of pre-defined class labels, the objective being to build a classifier to classify "unseen" data records. One recent approach to CRM is to employ Association Rule Mining (ARM) [1] methods to identify the desired CRs, i.e. Classification Association Rule Mining (CARM) [2]. CARM aims to mine a set of Classification Association Rules (CARs) from a class-transaction database, where a CAR describes an implicative co-occurring relationship between a set of binary-valued data attributes (items) and a pre-defined class, expressed in the form of an "〈antecedent $\rangle \Rightarrow\langle$ consequent-class〉" rule. CARM seems to offer greater accuracy, in many cases, than other classification methods such as decision trees, rule induction and probabilistic approaches [8] [13].

In the past decade, a number of CARM approaches have been developed that include: TFPC (Total From Partial Classification) [7] [8], CBA (Classification Based Associations) [11], CMAR (Classification based on Multiple Association Rules) [10], CPAR (Classification based on Predictive Association Rules) [15], etc. Although these CARM approaches employ different ARM techniques to extract CARs from a given class-transaction database, a similar set of CARs is always generated, based on a pair of specific values for both support and confidence thresholds. Regardless of which particular CARM method is utilized, a classifier is usually presented as an ordered list of CARs, based on a selected rule ordering strategy. Hence, it can be indicated that the essential to produce a more accurate CARM classifier is to develop a better (more rational) rule ordering approach.

Coenen and Leng [7] identify (1) three common CARM case satisfaction approaches: Best First Rule, Best $K$ Rules, and All Rules; and (2) five established CARM rule ordering mechanisms: Confidence Support size-of-rule-Antecedent (CSA), size-of-ruleAntecedent Confidence Support (ACS), Weighted Relative Accuracy (WRA), Laplace Accuracy (LAP), and Chi-square Testing $\left(\chi^{2}\right)$. Due to the sake of simplicity, the Best First Rule case satisfaction mechanism is widely applied in CARM classifiers. In [14] the authors divide the above rule ordering mechanisms into two groups: (type 1) supportconfidence based which includes CSA and ACS; and (type 2) rule weighting based which includes WRA, LAP and $\chi^{2}$. Wang et al. in [14] also propose a hybrid 
based ordering approach by combining one rule ordering mechanism taken from the (type 1) group and another rule ordering mechanism taken from the (type 2) group.

In this paper, we introduce a novel rule weighting scheme, namely CISRW (Class-Item Score based Rule Weighting), which assigns a weighting score to each CAR by assigning a weighting score to each CAR item. Then a rule ordering mechanism is proposed that simply sorts CARs in a descending order, based on their assigned CISRW score. As a consequence, two hybrid rule ordering strategies are further developed: (1) Hybrid CSA/CISRW, and (2) Hybrid ACS/CISRW. The experimental results show good performance regarding the accuracy of classification when using the proposed CISRW based/related rule ordering strategies with the Best First Rule case satisfaction.

The rest of this paper is organized as follows. Section 2 outlines the existing rule ordering strategies in CARM. The proposed rule weighting/ordering approach is described in section 3. In section 4 we present experimental results, and in section 5 our conclusions and open issues for further research.

\section{Overview of Rule Ordering Approaches}

\subsection{Support-Confidence Based Ordering}

- CSA: The CSA rule ordering strategy [11] sorts the CAR list in a descending order based on the value of conference of each CAR. For these CARs that share a common value of confidence, CSA sorts them in a descending order based on their support value. For these CARs that share common values for both confidence and support, CSA sorts them in an ascending order based on the size of the rule antecedent.

- ACS: The ACS rule ordering strategy [7] is a variation of CSA. It takes the size of the rule antecedent as its major factor (using a descending order) followed by the rule confidence and support values respectively.

\subsection{Rule Weighting Based Ordering}

- WRA: The WRA rule ordering strategy [9] assigns an additive weighting score to each CAR. The original CAR list is then sorted in a descending order based on the assigned scores. The calculation of the WRA score of a CAR $R$, confirmed in [7], is: support(R.antecedent) $\times$ (confidence $(R)$ - support(R.consequent-class)).

- LAP: The use of the Laplace Expected Error Estimate [5] can be found in [15]. The principle of applying this rule ordering mechanism is similar to WRA. The calculation of the LAP score of a CAR $R$ is: $(\operatorname{support}(R)+1) /(\operatorname{support}($ R.antecedent $)+$ $|C|$ ), where $|C|$ denotes the number of pre-defined classes.

- $\quad \chi^{2}$ : In $\chi^{2}$ Testing a set of observed values $O$ is compared against a set of expected values $E-$ values that would be estimated if there were no associative relationship between the variables. The value of $\chi^{2}$ is calculated as: $\sum_{\{i=1 \ldots n\}}\left(O_{i}-E_{i}\right)^{2} /$ $E_{i}$, where $n$ is the number of entries in the confusion matrix, which is always 4 in CARM. After assigning a $\chi^{2}$ value to each CAR, the original CAR list is then sorted in a descending order based on the assigned values.

\subsection{Hybrid Rule Ordering Schemes}

From the foregoing we can identify six hybrid rule ordering schemes [14]:

- Hybrid CSA/WRA: Selects the Best $K$ Rules (for each pre-defined class) in a WRA manner, and reorders both the best $K$ CAR list and the original CAR list in a CSA fashion. The best $K$ CAR list is linked at front of the original CAR list.

- Hybrid CSA/LAP: Selects the Best $K$ Rules (for each pre-defined class) in a LAP manner, and reorders both the best $K$ CAR list and the original CAR list in a CSA fashion. The best $K$ CAR list is linked at front of the original CAR list.

- Hybrid CSA $/ \chi^{2}$ : Selects the Best $K$ Rules (for each pre-defined class) in a $\chi^{2}$ manner, and reorders both the best $K$ CAR list and the original CAR list in a CSA fashion. The best $K$ CAR list is linked at front of the original CAR list.

- Hybrid ACS/WRA: Selects the Best $K$ Rules (for each pre-defined class) in a WRA manner, and reorders both the best $K$ CAR list and the original CAR list in an ACS fashion. The best $K$ CAR list is linked at front of the original CAR list.

- Hybrid ACS/LAP: Selects the Best $K$ Rules (for each pre-defined class) in a LAP manner, and reorders both the best $K$ CAR list and the original CAR list in an ACS fashion. The best $K$ CAR list is linked at front of the original CAR list.

- Hybrid ACS $/ \chi^{2}$ : Selects the Best $K$ Rules (for each pre-defined class) in a $\chi^{2}$ manner, and reorders both the best $K$ CAR list and the original CAR list in an ACS fashion. The best $K$ CAR list is linked at front of the original CAR list. 


\section{Proposed Rule Weighting/Ordering}

In this section, we describe the proposed CISRW rule weighting scheme, which assigns a weighting score to each CAR, by computing a score for each rule item and averaging the sum of all rule item scores. Then a rule weighting based rule ordering strategy is introduced founded on CISRW. As a consequence, two further hybrid rule ordering strategies that combining either CSA or ACS with CISRW, are proposed.

\subsection{Proposed Rule Weighting Scheme}

\subsubsection{Item Weighting Score}

There are $n$ items involved in a given classtransaction database $D_{C T}$ that is coupled with a set of pre-defined classes $C=\left\{c_{1}, c_{2}, \ldots, c_{m-1}, c_{m}\right\}$. For a particular pre-defined class $A$, a score is assigned to each item in $D_{C T}$ that distinguishes the significant items for class $A$ from the insignificant ones.

Definition 1. Let $C^{A}($ Item $)$ denote the contribution of each item ${ }_{h} \in D_{C T}$ for class $A$, which represents how

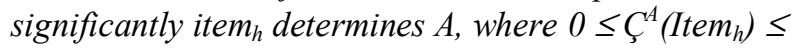
$|C|$, and $|C|$ is the size function of the set $C$.

The calculation of $C^{A}\left(\right.$ Item $\left._{h}\right)$ is: TransFreq $\left(\right.$ Item $\left._{h}, A\right)$

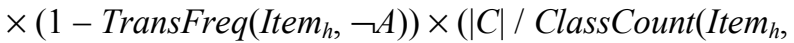
C)), where

- $\quad$ The TransFreq $\left(\right.$ Item $_{h}, A$ or $\left.\neg A\right)$ function computes how frequently that Item $_{h}$ appears in class $A$ or the group of classes $\neg A$ (the complement of $A$ ). The calculation of this function is: number of transactions with Item $_{h}$ in the class or class-group) I (number of transactions in the class or classgroup); and

- The Class Count $\left(\right.$ Item $\left._{h}, C\right)$ function simply counts the number of classes in $C$ which contain Item $_{h}$.

The rationale of this item weighing score is demonstrated as follows:

- The weighting score of Item $_{h}$ for class $A$ tends to be high if $\mathrm{Item}_{h}$ is frequent in $A$;

- The weighting score of Item $_{h}$ for class $A$ tends to be high if Item $_{h}$ is infrequent in $\neg A$;

- The weighting score of Item $_{h}$ for any class tends to be high if Item $_{h}$ is involved in a small number of classes in C. (In [4], a similar idea can be found in feature selection for text categorization.)

\subsubsection{Rule Weighting Score}

Based on the item weighting score, a rule weighing score is assigned to each CAR $R$ in the original CAR list.

Definition 2. Let $C^{A}(R)$ denote the contribution of each $C A R R$ in the original CAR list for class $A$ that represents how significantly $R$ determines $A$.

The calculation of $C^{A}(R)$ is: $\left(\sum_{\{h=1 \ldots \mid \text { R.antecedent } \mid\}}\right.$ $C^{A}\left(\right.$ Item $_{h} \in$ R.antecedent $\left.)\right) /(\mid$ R.antecedent $\mid)$, where $\mid R$.antecedent $\mid$ is the size function of the antecedent of this CAR.

\subsection{Proposed Rule Ordering Strategies}

- CISRW: The CISRW weighing score is assigned to each CAR, which represents how significantly the CAR antecedent determines its consequentclass. The original CAR list is then simply sorted in a descending order based on the assigned CISRW score of each CAR.

- Hybrid CSA/CISRW: Selects the Best $K$ Rules (for each pre-defined class) in a CISRW manner, and re-orders both the best $K$ CAR list and the original CAR list in a CSA fashion. The best $K$ CAR list is linked at front of the original CAR list.

- Hybrid ACS/CISRW: Selects the Best $K$ Rules (for each pre-defined class) in a CISRW manner, and re-orders both the best $K$ CAR list and the original CAR list in an ACS fashion. The best $K$ CAR list is linked at front of the original CAR list.

In Figure 1, a common procedure for both proposed Hybrid CSA/CISRW and Hybrid ACS/CISRW strategies is outlined.

Procedure Hybrid CSA(or ACS)/CISRW

Input: (a) A list of CARs $\mathfrak{i}$ (either in CSA or ACS ordering manner);

(b) A desired number (integer value) for the best rules $K$;

Output: A re-ordered list of CARs $9 i^{N E W}$ (either in Hybrid CSA/CISRW or Hybrid ACS/CISRW ordering manner);

(1) begin

(2) $\mathfrak{h}^{\mathrm{NEW}}:=\{\varnothing\}$;

(3) $\Re^{C I S R W}:=\{\varnothing\}$;

(4) for each $C A R \in \Re$ do

(5) calculate the CISRW score $(\delta)$ for this CAR;

(6) $g^{C I S R W} \leftarrow \mathfrak{i}^{C I S R W} \cup(\mathrm{CAR} \oplus \delta)$;

// the $\oplus$ sign means "with" an additive CAR attribute

(7) end for

(8) sort $\Re^{C I S R W}$ in a descending order based on $\delta$,

(9) $\mathfrak{R}^{\text {CISRW }} \leftarrow$ remain the top $K$ CARs (for each predefined class) $\in \mathfrak{S}^{C \text { CISRW }}$ 
(10) sort $\mathfrak{l}^{C I S R W}$ either in CSA or ACS ordering manner; // keep it consistent with $\Re$

(11) $\mathfrak{R}^{\mathrm{NEW}} \leftarrow$ link $\mathfrak{R}^{\mathrm{CISRW}}$ at front of $\mathfrak{R}$;

(12) return $\left(\Re^{N E W}\right)$;

(13) end

Figure 1. The Hybrid CSA(or ACS)/CISRW procedure

\section{Experimental Results}

In this section, we aim to evaluate the proposed CISRW based/related rule ordering strategies with respect to the accuracy of classification. All evaluations were obtained using the TFPC algorithm coupled with the Best First Rule case satisfaction, although any other CARM classifier generator, founded on the Best First Rule mechanism, could equally well be used. Experiments were run on a 1.86 GHz Intel(R) Core(TM)2 CPU with $1.00 \mathrm{~GB}$ of RAM running under Windows Command Processor.

The experiments were conducted using a range of datasets taken from the LUCS-KDD Discretised/Normalised ARM and CARM Data Library [6]. The chosen databases are originally taken from the UCI Machine Learning Repository [3]. These datasets have been discretized and normalized using the LUCS-KDD Discretised Normalised (DN) software, so that data are then presented in a binary format suitable for use with CARM applications. It should be noted that the datasets were re-arranged so that occurrences of classes were distributed evenly throughout. This then allowed the datasets to be divided in half with the first half used as the training set and the second half as the test set. Although a "better" accuracy figure might have been obtained using Ten-cross Validation, it is the relative accuracy that is of interest here and not the absolute accuracy.

The first set of evaluations undertaken used a confidence threshold value of $50 \%$ and a support threshold value $1 \%$ (as used in the published evaluations of CMAR [10], CPAR [15], TFPC [7] [8], and the hybrid rule ordering approach [14]). The results are presented in Table 1 where 120 classification accuracy values are listed based on 20 chosen datasets. The row labels describe the key characteristics of each dataset: for example, the label adult.D97.N48842.C2 denotes the "adult" dataset, which comprises 48,842 records in 2 pre-defined classes, with attributes that for the experiments described here have been discretized and normalized into 97 binary categories.

Table 1. Classification accuracy — support-confidence \& rule weighting strategies vs. the CISRW strategy

\begin{tabular}{|l|c|c|c|c|c|c|}
\hline DATASETS & CSA & ACS & WRA & LAP & $\chi^{2}$ & CISRW \\
\hline $\begin{array}{l}\text { adult.D97. } \\
\text { N48842.C2 }\end{array}$ & 80.83 & 73.99 & 81.66 & 76.07 & 76.07 & 81.61 \\
\hline
\end{tabular}

\begin{tabular}{|l|l|l|l|l|l|l|}
\hline $\begin{array}{l}\text { breast.D20. } \\
\text { N699.C2 }\end{array}$ & 89.11 & 89.11 & 87.68 & 65.62 & 65.62 & 87.68 \\
\hline $\begin{array}{l}\text { chessKRvK.D58 } \\
\text {.N28056.C18 }\end{array}$ & 14.95 & 14.95 & 14.95 & 14.95 & 14.95 & 14.95 \\
\hline $\begin{array}{l}\text { connect4.D129. } \\
\text { N67557.C3 }\end{array}$ & 65.83 & 64.83 & 67.93 & 65.83 & 65.83 & 66.94 \\
\hline $\begin{array}{l}\text { flare.D39. } \\
\text { N.1389.C9 }\end{array}$ & 84.44 & 83.86 & 84.15 & 84.44 & 84.44 & 84.44 \\
\hline $\begin{array}{l}\text { glass.D48. } \\
\text { N214.C7 }\end{array}$ & 58.88 & 43.93 & 50.47 & 52.34 & 50.47 & 55.14 \\
\hline $\begin{array}{l}\text { heart.D52. } \\
\text { N303.C5 }\end{array}$ & 58.28 & 28.48 & 55.63 & 54.97 & 54.97 & 57.62 \\
\hline $\begin{array}{l}\text { horseColic. } \\
\text { D85.N368.C2 }\end{array}$ & 72.83 & 40.76 & 79.89 & 79.89 & 63.04 & 79.89 \\
\hline $\begin{array}{l}\text { ionosphere. } \\
\text { D157.N351.C2 }\end{array}$ & 85.14 & 61.14 & 86.86 & 64.57 & 64.57 & 83.43 \\
\hline $\begin{array}{l}\text { iris.D19. } \\
\text { N.150.C3 }\end{array}$ & 97.33 & 97.33 & 97.33 & 97.33 & 97.33 & 97.33 \\
\hline $\begin{array}{l}\text { led7.D24. } \\
\text { N3200.C10 }\end{array}$ & 68.38 & 61.38 & 63.94 & 63.88 & 66.56 & 60.50 \\
\hline $\begin{array}{l}\text { letRecog.D106. } \\
\text { N20000.C26 }\end{array}$ & 31.13 & 26.21 & 26.33 & 26.33 & 28.52 & 26.38 \\
\hline $\begin{array}{l}\text { mushroom.D90. } \\
\text { N8124.C2 }\end{array}$ & 99.21 & 65.76 & 98.45 & 98.45 & 49.43 & 98.45 \\
\hline $\begin{array}{l}\text { nursery.D32. } \\
\text { N12960.C5 }\end{array}$ & 80.35 & 55.88 & 70.17 & 70.17 & 70.17 & 70.17 \\
\hline $\begin{array}{l}\text { pageBlocks.D46. } \\
\text { N5473.C55 }\end{array}$ & 90.97 & 90.97 & 90.20 & 89.80 & 89.80 & 91.56 \\
\hline $\begin{array}{l}\text { pima..338. } \\
\text { N768.C2 }\end{array}$ & 73.18 & 71.88 & 72.92 & 65.10 & 65.10 & 72.92 \\
\hline $\begin{array}{l}\text { soybean-large. } \\
\text { D118.N683.C19 }\end{array}$ & 86.22 & 79.77 & 36.36 & 36.07 & 77.42 & 63.93 \\
\hline $\begin{array}{l}\text { ticTTacToe.D29. } \\
\text { N958.C2 }\end{array}$ & 71.61 & 36.12 & 68.06 & 65.34 & 65.34 & 68.27 \\
\hline $\begin{array}{l}\text { waveform.D101 } \\
\text { N5000.C3 }\end{array}$ & 61.56 & 47.96 & 56.24 & 57.84 & 57.28 & 56.08 \\
\hline $\begin{array}{l}\text { zoo.D42. } \\
\text { N101.C7 }\end{array}$ & 80.00 & 42.00 & 56.00 & 42.00 & 42.00 & 86.00 \\
\hline Average & $\mathbf{7 2 . 5 1}$ & $\mathbf{5 8 . 8 2}$ & $\mathbf{6 7 . 2 6}$ & $\mathbf{6 3 . 5 5}$ & $\mathbf{6 2 . 4 5}$ & $\mathbf{7 0 . 1 6}$ \\
\hline
\end{tabular}

From Table 1 it is clear that with a $50 \%$ confidence threshold and a 1\% support threshold the CSA mechanism worked better than other alternative nonhybrid rule ordering strategies. When applying CSA, the average accuracy of classification throughout the 20 datasets is $72.51 \%$. The performance rank of the five established rule ordering mechanisms is specified as follows: (1) CSA - the average accuracy of classification is $72.51 \%$; (2) WRA - the accuracy is $67.26 \%$; (3) LAP $-63.55 \%$; (4) $\chi^{2}-62.45 \%$; and (5) ACS - 58.82\%. It should be noted that this ranking result corroborates to the results presented in [14] although both investigations involve different datasets in their experimentation section. With respect to the group of rule weighting based rule ordering mechanisms, the proposed CISRW performed better than other rule weighting mechanisms, where its average accuracy of classification throughout the 20 datasets is $70.16 \%$.

The second set of evaluations undertaken used a confidence threshold value of $50 \%$, a support threshold value of $1 \%$, and a value of 5 as an appropriate value for $K$ when selecting the Best $K$ Rules $(K=5$ was also used in [15]). The results are demonstrated in Table 2 where 80 classification accuracy values are listed based on 20 chosen datasets.

Table 2. Classification accuracy — CSA based hybrid strategies vs. the Hybrid CSA/CISRW strategy

\begin{tabular}{|l|c|c|c|c|}
\hline DATASETS & $\begin{array}{c}\text { Hybrid } \\
\text { CSA/WRA }\end{array}$ & $\begin{array}{c}\text { Hybrid } \\
\text { CSA/LAP }\end{array}$ & $\begin{array}{c}\text { Hybrid } \\
\text { CSA } / \chi^{2}\end{array}$ & $\begin{array}{c}\text { Hybrid } \\
\text { CSA/CISRW }\end{array}$ \\
\hline
\end{tabular}




\begin{tabular}{|l|l|l|l|l|}
\hline $\begin{array}{l}\text { adult.D97. } \\
\text { N48842.C2 }\end{array}$ & 83.33 & 79.95 & 79.95 & 81.46 \\
\hline $\begin{array}{l}\text { breast.D20. } \\
\text { N699.C2 }\end{array}$ & 89.11 & 88.54 & 89.11 & 89.11 \\
\hline $\begin{array}{l}\text { chessKRvK.D58. } \\
\text { N28056.C18 }\end{array}$ & 14.95 & 14.95 & 14.95 & 14.95 \\
\hline $\begin{array}{l}\text { connect4.D129. } \\
\text { N67557.C3 }\end{array}$ & 67.67 & 65.83 & 65.83 & 66.71 \\
\hline $\begin{array}{l}\text { flare.D39. } \\
\text { N.1389.C9 }\end{array}$ & 84.29 & 54.44 & 84.44 & 84.15 \\
\hline $\begin{array}{l}\text { glass.D48. } \\
\text { N214.C7 }\end{array}$ & 66.36 & 66.36 & 66.36 & 65.42 \\
\hline $\begin{array}{l}\text { heart.D52. } \\
\text { N303.C5 }\end{array}$ & 55.63 & 56.95 & 58.94 & 58.28 \\
\hline $\begin{array}{l}\text { horseColic. } \\
\text { D85.N368.C2 }\end{array}$ & 83.15 & 83.15 & 79.89 & 83.15 \\
\hline $\begin{array}{l}\text { ionosphere. } \\
\text { D157.N351.C2 }\end{array}$ & 90.29 & 89.71 & 88.00 & 89.14 \\
\hline $\begin{array}{l}\text { iris.D19. } \\
\text { N.150.C3 }\end{array}$ & 97.33 & 97.33 & 97.33 & 97.33 \\
\hline $\begin{array}{l}\text { led7.D24. } \\
\text { N3200.C10 }\end{array}$ & 68.19 & 68.19 & 68.38 & 68.38 \\
\hline $\begin{array}{l}\text { letRecog.D106. } \\
\text { N20000.C26 }\end{array}$ & 31.49 & 31.49 & 31.56 & 30.87 \\
\hline $\begin{array}{l}\text { mushroom.D90. } \\
\text { N8124.C2 }\end{array}$ & 98.45 & 98.82 & 98.45 & 98.82 \\
\hline $\begin{array}{l}\text { nursery.D32. } \\
\text { N12960.C5 }\end{array}$ & 78.86 & 78.86 & 78.86 & 78.26 \\
\hline $\begin{array}{l}\text { pageBlocks.D46. } \\
\text { N5473.C5 }\end{array}$ & 90.97 & 90.97 & 90.97 & 90.97 \\
\hline $\begin{array}{l}\text { pima.D38. } \\
\text { N768.C2 }\end{array}$ & 73.18 & 73.18 & 72.66 & 73.44 \\
\hline $\begin{array}{l}\text { soybean-large. } \\
\text { D118.N683.C19 }\end{array}$ & 80.94 & 80.94 & 82.11 & 83.58 \\
\hline $\begin{array}{l}\text { ticTacToe.D29. } \\
\text { N958.C2 }\end{array}$ & 74.95 & 74.74 & 72.65 & 73.90 \\
\hline $\begin{array}{l}\text { waveform.D101. } \\
\text { N5000.C3 }\end{array}$ & 57.96 & 57.96 & 60.60 & 58.40 \\
\hline $\begin{array}{l}\text { zoo.D42. } \\
\text { N101.C7 }\end{array}$ & 84.00 & 90.00 & 72.00 & 88.00 \\
\hline Average & $\mathbf{7 3 . 5 6}$ & $\mathbf{7 3 . 6 2}$ & $\mathbf{7 2 . 6 5}$ & $\mathbf{7 3 . 7 2}$ \\
\hline
\end{tabular}

\begin{tabular}{|l|l|l|l|l|}
\hline $\begin{array}{l}\text { flare.D39. } \\
\text { N.1389.C9 }\end{array}$ & 83.86 & 83.86 & 83.86 & 83.86 \\
\hline $\begin{array}{l}\text { glass.D48. } \\
\text { N214.C7 }\end{array}$ & 65.42 & 65.42 & 68.22 & 63.55 \\
\hline $\begin{array}{l}\text { heart.D52. } \\
\text { N303.C5 }\end{array}$ & 52.32 & 50.33 & 50.33 & 52.32 \\
\hline $\begin{array}{l}\text { horseColic. } \\
\text { D85.N368.C2 }\end{array}$ & 75.00 & 83.15 & 71.20 & 78.80 \\
\hline $\begin{array}{l}\text { ionosphere. } \\
\text { D157.N351.C2 }\end{array}$ & 90.29 & 89.71 & 88.00 & 89.14 \\
\hline $\begin{array}{l}\text { iris.D19. } \\
\text { N.150.C3 }\end{array}$ & 97.33 & 97.33 & 97.33 & 97.33 \\
\hline $\begin{array}{l}\text { led7.D24. } \\
\text { N3200.C10 }\end{array}$ & 62.06 & 62.06 & 62.31 & 65.69 \\
\hline $\begin{array}{l}\text { letRecog.D106. } \\
\text { N20000.C26 }\end{array}$ & 27.39 & 27.39 & 28.41 & 28.58 \\
\hline $\begin{array}{l}\text { mushroom.D90. } \\
\text { N8124.C2 }\end{array}$ & 98.45 & 98.82 & 98.45 & 98.82 \\
\hline $\begin{array}{l}\text { nursery.D32. } \\
\text { N12960.C5 }\end{array}$ & 66.73 & 66.73 & 66.73 & 71.28 \\
\hline $\begin{array}{l}\text { pageBlocks.D46. } \\
\text { N5473.C5 }\end{array}$ & 90.97 & 90.97 & 90.97 & 90.97 \\
\hline $\begin{array}{l}\text { pima.D38. } \\
\text { N768.C2 }\end{array}$ & 73.18 & 73.18 & 72.66 & 71.61 \\
\hline $\begin{array}{l}\text { soybean-large. } \\
\text { D118.N683.C19 }\end{array}$ & 75.66 & 75.66 & 78.01 & 78.59 \\
\hline $\begin{array}{l}\text { ticTacToe.D29. } \\
\text { N958.C2 }\end{array}$ & 60.75 & 70.35 & 67.22 & 67.22 \\
\hline $\begin{array}{l}\text { waveform.D101. } \\
\text { N5000.C3 }\end{array}$ & 59.20 & 59.20 & 60.60 & 58.40 \\
\hline $\begin{array}{l}\text { zoo.D42. } \\
\text { N101.C7 }\end{array}$ & 80.00 & 80.00 & 80.00 & 76.00 \\
\hline Average & $\mathbf{7 0 . 3 1}$ & $\mathbf{7 1 . 3 1}$ & $\mathbf{7 0 . 6 7}$ & $\mathbf{7 1 . 1 5}$ \\
\hline
\end{tabular}

From Table 3 it can be seen that with a $50 \%$ confidence threshold, a $1 \%$ support threshold, and $K=$ 5 , the best-performing hybrid ACS related rule ordering strategy is the Hybrid ACS/LAP mechanism. When applying this mechanism, the average accuracy of classification throughout the 20 datasets is $71.31 \%$. The proposed Hybrid ACS/CISRW approach demonstrated a comparable performance to Hybrid ACS/LAP, where its average classification accuracy is $71.15 \%$. The performances of three existing ACS related hybrid strategies were ranked as: (1) ACS/LAP - 71.31\%; (2) $\mathrm{ACS} / \chi^{2}$ - the average accuracy is $70.67 \%$; and (3) ACS/WRA - 70.31\%. This ranking result corroborates to the experimental results provided in [14] although different datasets were concerned.

\section{Conclusion}

CARM is a recent CRM approach that aims to classify "unseen" data based on building an ARM based classifier. A number of literatures have confirmed the outstanding performance of CARM. It can be clarified that no matter which particular ARM technique is employed, a similar set of CARs is always generated from data, and a classifier is usually presented as an ordered list of CARs, based on an applied rule ordering strategy. In this paper a novel rule weighting approach was proposed, which assigns a weighting score to each generated CAR. Based on the proposed rule weighting approach, a straightforward rule ordering mechanism (CISRW) was introduced. Subsequently, two hybrid rule ordering strategies 
(Hybrid CSA/CISRW and Hybrid ACS/CISRW) were further developed.

Table 4. Ranking of rule ordering strategies

\begin{tabular}{|c|c|c|c|}
\hline Rank No. & Rule Ordering Strategy & $\begin{array}{c}\text { Average } \\
\text { Accuracy }\end{array}$ & $\begin{array}{c}\text { Rank No. } \\
\text { in [14] }\end{array}$ \\
\hline $\mathbf{1}$ & Hybrid CSA/CISRW & 73.72 & - \\
\hline $\mathbf{2}$ & Hybrid CSA/LAP & 73.62 & $\mathbf{1}$ \\
\hline $\mathbf{3}$ & Hybrid CSA/WRA & 73.56 & $\mathbf{2}$ \\
\hline $\mathbf{4}$ & Hybrid CSA/ $\chi^{2}$ & 72.65 & $\mathbf{3}$ \\
\hline $\mathbf{5}$ & CSA & 72.51 & $\mathbf{4}$ \\
\hline $\mathbf{6}$ & Hybrid ACS/LAP & 71.31 & $\mathbf{5}$ \\
\hline $\mathbf{7}$ & Hybrid ACS/CISRW & 71.15 & - \\
\hline $\mathbf{8}$ & Hybrid ACS/ $\chi^{2}$ & 70.67 & $\mathbf{6}$ \\
\hline $\mathbf{9}$ & Hybrid ACS/WRA & 70.31 & $\mathbf{7}$ \\
\hline $\mathbf{1 0}$ & CISRW & 70.16 & - \\
\hline $\mathbf{1 1}$ & WRA & 67.26 & $\mathbf{8}$ \\
\hline $\mathbf{1 2}$ & LAP & 63.55 & $\mathbf{9}$ \\
\hline $\mathbf{1 3}$ & $\chi$ & 62.45 & $\mathbf{1 0}$ \\
\hline $\mathbf{1 4}$ & ACS & 58.82 & $\mathbf{1 1}$ \\
\hline
\end{tabular}

From the experimental results, it can be seen that the average accuracy of classification, using the 20 chosen datasets, obtained by the proposed Hybrid CSA/CISRW rule ordering strategy can be better than other alternative rule ordering mechanisms, where the accuracy is $73.72 \%$. In Table 4 , the rank of classification accuracies for all fourteen rule ordering strategies is presented. The proposed Hybrid CSA/CISRW, Hybrid ACS/CISRW and CISRW rule ordering mechanisms were ranked as No.1, No. 7 and No. 10. Furthermore the performances of eleven existing rule ordering strategies were ranked in accordance with the results presented in [14] although different datasets were used in both investigations.

Further research is suggested to identify the improved rule weighting/ordering approach in CARM to give a better performance.

\section{Acknowledgments}

The authors would like to thank Prof. Paul Leng and Dr. Robert Sanderson of the Department of Computer Science at the University of Liverpool for their support with respect to the work described here.

\section{References}

[1] R. Agrawal and R. Srikant, "Fast Algorithm for Mining Association Rules", In Proceedings of the $20^{\text {th }}$ International Conference on Very Large Data Bases (VLDB-94), Morgan Kaufmann Publishers, Santiago de Chile, Chile, September 1994, pp. 487-499.

[2] K. Ali, S. Manganaris, and R. Srikant, "Partial Classification using Association Rules", In Proceedings of the $3^{\text {rd }}$ International Conference on Knowledge Discovery and Data Mining (KDD97), AAAI Press, Newport Beach, California, United States, August 1997, pp. 115-118.

[3] C.L. Blake and C.J. Merz, "UCI Repository of Machine Learning Databases", Department of Information and Computer
Science, University of California, Irvine, CA, United States, 1998. http://www.ics.uci.edu/ mlearn/MLRepository.html

[4] C.H. Bong and K. Narayanan, "An Empirical Study of Feature Selection for Text Categorization based on Term Weightage", In Proceedings of the 2004 IEEE/WIC/ACM International Conference on Web Intelligence (WI-04), IEEE Computer Society, Beijing, China, September 2004, pp. 599-602.

[5] P. Clark and R. Boswell, "Rule Induction with CN2: Some Recent Improvement", In Proceedings of the Fifth European Working Session on Learning (EWSL-91), Springer-Verlag, Porto, Portugal, March 1991, pp. 111-116.

[6] F. Coenen, "The LUCS-KDD Discretised/Normalised ARM and CARM Data Library", Department of Computer Science, The University of Liverpool, Liverpool, United Kingdom, 2003. http://www.csc.liv.ac.uk/ frans/KDD/Software/LUCS-KDD-DN

[7] F. Coenen and P. Leng, "An Evaluation of Approaches to Classification Rule Selection", In Proceedings of the $4^{\text {th }}$ IEEE International Conference on Data Mining (ICDM-04), IEEE Computer Society, Brighton, United Kingdom, November 2004, pp. 359-362.

[8] F. Coenen, P. Leng, and L. Zhang, "Threshold Tuning for Improved Classification Association Rule Mining", In Proceedings of the $9^{\text {th }}$ Pacific-Asia Conference on Knowledge Discovery and Data Mining (PAKDD-05), Springer-Verlag, Hanoi, Vietnam, 2005, pp. 216-225.

[9] N. Lavrac, P. Flach, and B. Zupan, "Rule Evaluation Measures: A Unifying View", In Proceedings of the $9^{\text {th }}$ International Workshop on Inductive Logic Programming (ILP99), Springer-Verlag, Bled, Slovenia, June 1999, pp. 174-185.

[10] W. Li, J. Han, and J. Pei, "CMAR: Accurate and Efficient Classification based on Multiple Class-association Rules", In Proceedings of the 2001 IEEE International Conference on Data Mining (ICDM-01), IEEE Computer Society, San Jose, California, United States, 2001, pp. 369-376.

[11] B. Liu, W. Hsu, and Y. Ma, "Integrating Classification and Association Rule Mining", In Proceedings of the $4^{\text {th }}$ International Conference on Knowledge Discovery and Data Mining (KDD-98), AAAI Press, New York City, New York, United States, 1998, pp. 80-86.

[12] Quinlan, J.R., C4.5: Programs for Machine Learning, Morgan Kaufmann Publishers, San Mateo, CA, United States, 1993.

[13] F. Thabtah, P. Cowling, and Y. Peng, "The Impact of Rule Ranking on the Quality of Associative Classifiers", In Proceedings of AI-2005, the Twenty-fifth SGAI International Conference on Innovative Techniques and Applications of Artificial Intelligence (AI-2005), Springer-Verlag, Cambridge, United Kingdom, December 2005, pp. 277-287.

[14] Y.J. Wang, Q. Xin, and F. Coenen, "A Novel Rule Ordering Approach in Classification Association Rule Mining", In Proceedings of the $5^{\text {th }}$ International Conference on Machine Learning and Data Mining (MLDM-07), Springer-Verlag, Leipzig, Germany, July 2007, pp. 339-348.

[15] X. Yin and J. Han, "CPAR: Classification based on Predictive Association Rules", In Proceedings of the Third SIAM International Conference on Data Mining (SDM-03), SIAM, San Francisco, CA, United States, 2003, pp. 331-335. 